\begin{tabular}{c|c|c|}
$\begin{array}{c}\text { SELECCIONES MATEMÁTICAS } \\
\text { Universidad Nacional de Trujillo } \\
\text { ISSN: } 2411-1783 \text { (Online) } \\
\text { 2021; Vol. 8(2): } 267-273 .\end{array}$ \\
\hline
\end{tabular}

\title{
Periodicity in a Gause-type predation model considering collaboration among predators
}

\section{Periodicidad en modelos de depredación del tipo Gause considerando colaboración entre los depredadores}

\author{
Eduardo González-Olivares ${ }^{\circledR}$ and Alejandro Rojas-Palma (i)
}

Received, Set. 10, 2021

Accepted, Nov. 03, 2021

How to cite this article:

González-Olivares E, Rojas-Palma A. Periodicity in a Gause-type predation model considering collaboration among predators. Selecciones Matemáticas. 2021;8(2):267-273. http://dx.doi.org/10.17268/sel.mat.

2021.02 .05

\section{Creative Commons - Attribution 4.0 International (CC BY 4.0)}

\section{Abstract}

Predation models are a great source of study from both an ecological and a mathematical point of view, especially for the analysis of trophic chains.

The determination of the dynamics of the systems that describe them, as well as the verification of the nature of these properties by the interacting species, is a topic that is sometimes not always correlated.

It is widely known that the incorporation of some mathematical descriptions of ecological phenomena strongly modifies the properties of many of these models. This implies that the systems describing such models are structurally unstable.

In this work, we include collaboration or cooperation between predators, a social behavior that describes the help made to capture their favorite prey. It is described by a power function with an exponent between 0 and 1, to indicate the possible interference between them, despite their collaboration. The exponent is interpreted as the density-dependent aggregation index.

We show that this assumption originates a varied behavior of the system, with respect to the associated Kolmogorov-type quadratic polynomial system that does not consider collaboration, including the existence of a stable limit cycle around a positive equilibrium point, among other analytical properties.

Keywords . Predator-prey model, functional response, cooperation, stability, bifurcations, limit cycles.

\section{Resumen}

Los modelos de depredación constituyen una gran fuente de estudio tanto del punto de vista ecológico como matemático, en especial para el análisis de cadenas tróficas.

La determinación de las dinámicas de los sistemas que los describen, como la comprobación en la naturaleza de estas propiedades por las especies interactuantes, es un tópico que a veces no siempre está correlacionado.

Es ampliamente sabido que la incorporación de algunas descripciones matemáticas de fenómenos ecológicos, modifican fuertemente las propiedades de muchos de esos modelos. Esto implica que los sistemas describiendo tales modelos son estructuralmente inestables.

En este trabajo incluimos la colaboración o cooperación entre los depredadores, un comportamiento social que describe la ayuda realizada para la captura de su presa favorita. Es descrito por una función potencia con exponente entre 0 y 1, para indicar la posible interferencia entre ellos, a pesar de su colaboración. El exponente se interpreta como el índice de agregación dependiente de la densidad.

\footnotetext{
${ }^{*}$ Pontificia Universidad Católica de Valparaíso, Chile. (e jgonzal@ucv.cl).

${ }^{\dagger}$ Departamento de Matemática, Física y Estadística, Facultad de Ciencias Básicas, Universidad Católica del Maule, Talca, Chile. (amrojas@ucm.cl)
} 
Mostramos que este supuesto origina un variado comportamiento del sistema, respecto al sistema de polinomial cuadrático del tipo Kolmogorov asociado que no considera colaboración, incluyendo la existencia de un ciclo límite estable alrededor de un punto de equilibrio positivo, entre otras propiedades analíticas.

Palabras clave. Modelo depredador-presa, respuesta funcional, colaboración, estabilidad, bifurcaciones, ciclo límite.

1. Introducción. Usualmente, el analisis de los modelos depredador-presa considera diferentes fenómenos ecológicos que afectan a una o ambas poblaciones. Estos fenómenos pueden tener fuertes consecuencias en la relación entre las especies y en la modificación de las propiedades dinámicas de los sistemas que lo describen. Tal es el caso de la colaboración o de la competición entre depredadores (CAP) [12].

La colaboración o cooperación (hunting cooperation) entre los depredadores para capturar sus presas es un comportamiento social que está recibiendo una creciente atención de los modeladores [17, 24].

Existen variadas formulaciones matemáticas para describir la colaboración entre los depredadores [4]. En este trabajo consideraremos un modelo simple propuesto por P. L. Antonelli y N. D. Kazarinoff en [3], el cual modela la depredación de corales por un tipo de estrella de mar, las cuales actuan en grupo para consumir sus presas [1,2]. Otros ejemplos de colaboración entre los depredadores registrados y considerados en literatura ecológica son: los lobos que siguen a los bisontes [19], las hienas que persiguen a los búfalos, los perros salvajes africanos (lincaones) en busca de cebras [16], entre otros.

En este trabajo incorporaremos esta interacción entre los depredadores en un modelo del tipo Gause [8], nombre dado en honor del biologo ruso Georgii F. Gause [9]. Este tipo de modelos ha sido la forma más común para modelar a depredadores especialistas (modelos recurso-consumidor), siendo el más conocido y más antiguo el modelo Lotka-Volterra, el "abuelo" de los modelos de depredación [18].

La característica principal de estos modelos es que son sistemas compartimentados, basados en un principio de transferencia de masa o energía [5].

Para modelar la colaboración usaremos una función potencia descrita por $h(y)=e y^{\sigma}$, con $e>0 \mathrm{y}$ $0<\sigma<1$, la cual es dependiente sólo de a población de depredadores [16].. Se adiciona a la segunda ecuación del modelo de Volterra [25], que es una modificación del modelo de Lotka-Volterra asumiendo crecimiento lógistico en la población de presas [20].

Sin embargo, otras funciones pueden ser consideradas para describir la colaboración entre los depredadores; por ejemplo considerando funciones saturadas [24, 26]. Estas funciones dan origen a nuevos modelos que podrán ser abordados en un trabajo futuro.

Este artículo está organizado de la siguiente manera: En la Sección 2 proponemos el modelo a estudiar; en la Sección 3 establecemos sus propiedades fundamentales. En la última Sección 4 establecemos las principales implicaciones ecológicas de los resultados matemáticos.

2. Proposición del modelo. El modelo analizado es derivado del modelo de Volterra [25], el cual a su vez es derivado del muy conocido modelo de Lotka-Volterra [4, 20], agregando un nuevo término en la ecuación de crecimiento de los depredadores. Es descrito por el siguiente sistema de EDO autónomas del tipo Kolmogorov [8, 10, 15]:

$$
X_{\nu}(x, y):\left\{\begin{aligned}
\frac{d x}{d t} & =\left(r\left(1-\frac{x}{K}\right)-q y\right) x \\
\frac{d y}{d t} & =\left(p x-c+e y^{\sigma}\right) y
\end{aligned}\right.
$$

donde $x=x(t)$ e $y=y(t)$ indican los tamaños de la población de presas y depredadores, respectivamente para $t \geq 0$, medido como biomasa o densidad por unidad de área o volumen.

Los parámetros son todos positivos, es decir, $\left.\nu=(r, K, q, p, c, e, \sigma) \in \mathbb{R}_{+}^{6} \times\right] 0,1[$, teniendo diferentes significados ecológicos.

El sistema (2.1) está definido en todo el primer cuadrante, es decir:

$$
\Omega=\left\{(x, y) \in \mathbb{R}^{2} / x \geq 0, y \geq 0\right\}=\mathbb{R}_{0}^{+} \times \mathbb{R}_{0}^{+} .
$$

Los puntos de equilibrio del sistema son $(0,0),(K, 0),\left(0,\left(\frac{c}{e}\right)^{\frac{1}{\sigma}}\right)$ y $\left(x_{e}, y_{e}\right)$ que satisface la ecuación de las nulclinas $y=\frac{r}{q}\left(1-\frac{x}{K}\right)$ e $y=\left(\frac{1}{e}(p x-c)\right)^{\frac{1}{\sigma}}$.

\section{Observación 2.1.}

Claramente, el valor del parámetro $\sigma$ influye en la dinámica del modelo que considera cooperación entre depredadores. 
i) Si $\sigma=0$, el sistema anterior se reduce al clásico modelo de Volterra [25], un caso especial del modelo de Gause [9] con crecimiento logístico de la presa y respuesta funcional lineal (que NO es de Holling tipo I), cuya dinámica es bastante conocida [15].

ii) Si $\sigma=1$, el sistema fue estudiado en [13] el cual tiene algunas de las dinámicas que se muestran en este trabajo.

iii) cuando $\sigma>1$, la dinámica del sistema es similar al modelo estudiado en [13].

iv) Cuando $0<\sigma<1$, el sistema no está estudiado completamente y la descripción de su dinámica será el objetivo principal del presente trabajo.

Para simplificar los cálculos, seguimos la metodología utilizada en [12, 13], haciendo un cambio de variables y reescalando el tiempo, lo cual es descrito en la siguiente:

Proposición 2.1. Sistema topológicamente equivalente El campo vectorial $X_{\nu}(x, y)$ o sistema $(2,1)$ topológicamente equivalente a

$$
Y_{\mu}(u, v):\left\{\begin{aligned}
\frac{d u}{d \tau} & =(1-u-v) u \\
\frac{d v}{d \tau} & =B\left(u-C+N v^{\sigma}\right) v
\end{aligned}\right.
$$

$\left.\operatorname{con} \mu=(B, C, N, \sigma) \in \mathbb{R}_{+}^{3} \times\right] 0,1\left[\right.$ donde $B=\frac{p K}{r}, C=\frac{c}{p K}$ y $N=\frac{e}{p K}\left(\frac{r}{q}\right)^{\sigma}$.

Demostración: Considerando el difeomorfismo $\phi(u, v, \tau)=\left(K u, \frac{r}{q} v, r \tau\right)$, que preserva la orientación del tiempo.

El sistema (2.2) o campo vectorial $Y_{\mu}(u, v)$ está también definido en todo el primer cuadrante, es decir: $\bar{\Omega}=\left\{(u, v) \in \mathbb{R}^{2} / u \geq 0, v \geq 0\right\}=\mathbb{R}_{0}^{+} \times \mathbb{R}_{0}^{+}$.

Los puntos de equilibrio del sistema $(2.2)$, son $(0,0),(1,0),\left(0,\left(\frac{C}{N}\right)^{\frac{1}{\sigma}}\right)$ y los que está en la intersección de las isoclinas $v=1-u \mathrm{y} v=\left(\frac{C-u}{N}\right)^{\frac{1}{\sigma}}$.

\section{Proposición 2.2.}

Existe un único punto de equilibrio positivo $\left(u_{e}, v_{e}\right)$, si y sólo si,

i) $1<C$ y $\frac{C}{N}<1$, o bien,

ii) $1>C$ y $\frac{C}{N}>1$.

Demostración: La isoclina de las presas $v=1-u$ es una recta decreciente que pasa por los puntos $(0,1)$ y $(1,0)$.

La isoclina de los depredadores $v=\left(\frac{C-u}{N}\right)^{\frac{1}{\sigma}}$ es una curva decreciente que pasa por los puntos $\left(0,\left(\frac{C}{N}\right)^{\frac{1}{\sigma}}\right)$ y $(C, 0)$. Está definida, si y sólo si, $C>u$.

La derivada de la isoclina de los depredadores es

$$
\frac{d}{d u}\left(\left(\frac{C-u}{N}\right)^{\frac{1}{\sigma}}\right)=-\frac{1}{N \sigma}\left(\frac{1}{N}(C-u)\right)^{\frac{1-\sigma}{\sigma}}<0 \text {, si y sólo si, } C>u .
$$

Las isoclinas intersectan, si y sólo si, $\left(\frac{C}{N}\right)^{\frac{1}{\sigma}}<1$ y $C>1$, o bien, $\left(\frac{C}{N}\right)^{\frac{1}{\sigma}}>1$ y $C<1$.

Luego, las isoclinas se intersectan al interior del primer cuadrante, si y sólo si, i) $1<C$ y $\frac{C}{N}<1$, o bien, ii) $1>C$ y $\frac{C}{N}>1$.

Notamos que si $\frac{C}{N}<1$ y $C<1$, o bien, si $\frac{C}{N}>1$ y $C>1$, no hay intersección entre las isoclinas.

En la Figura 2.1, se muestran los gráficos de las isoclinas para valores de $\sigma$ y $N$ fijos, con diferentes valores del parámetro $C$.

Notamos que la abscisa $u_{e}$ de los puntos de equilibrio interiores del primer cuadrante o positivo, satisface la ecuación trascendente:

$$
p(u)=u-C+N(1-u)^{\sigma}=0 .
$$

La matriz Jacobiana es

$$
D Y_{\mu}(u, v)=\left(\begin{array}{cc}
1-v-2 u & -u \\
B v & B\left(u-C+(1+\sigma) N v^{\sigma}\right)
\end{array}\right),
$$

la cual esta definida para todo punto $(u, v) \in \bar{\Omega}$. 


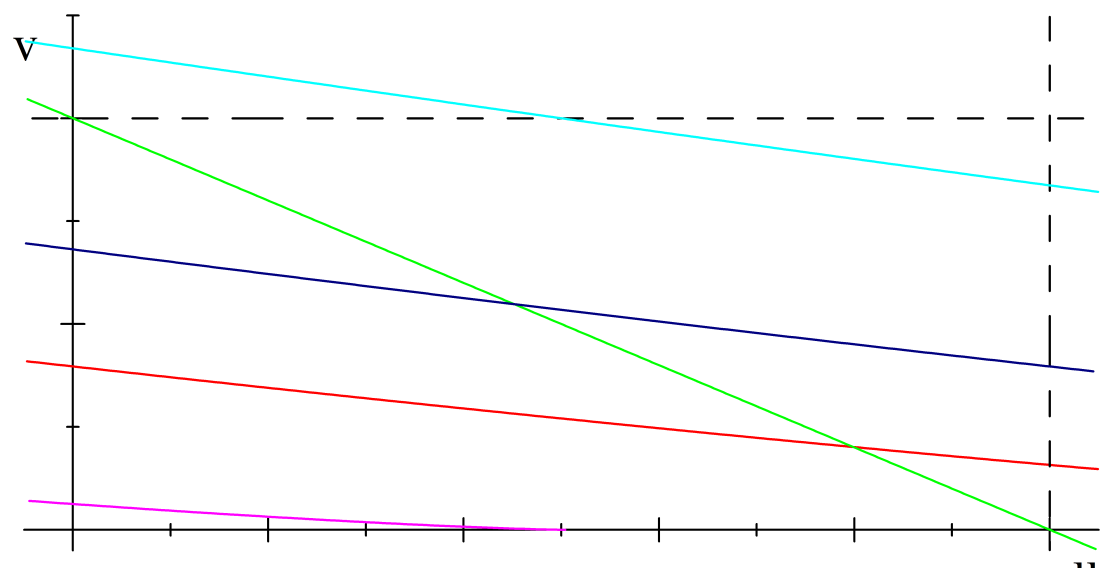

Figura 2.1: Isoclinas obtenidas para $C=0,5$ (magenta), 0,85 (sienna), 2 (rojo), 3 (azul), 4,5 (celeste) con $N=4$ y $\sigma=0,75$ fijos y la recta $v=1-u$ en verde.

3. Resultados principales. Asumiendo que $0<\sigma<1$, para el sistema (2.2) o campo vectorial $Y_{\mu}(u, v)$ se tienen las siguientes propiedades:

Proposición 3.1. Existencia de una región positivamente invariante.

El conjunto $\bar{\Gamma}=\left\{(u, v) \in \mathbb{R}^{2}: 0 \leq u \leq 1, v \geq 0\right\} \subset \bar{\Omega}$ es una región positivamente invariante.

Demostración: El sistema (2.2) es de tipo Kolmogorov [8, 10, 15] y los ejes son conjuntos invariantes.

Asumiendo $u=1$, se tiene que:

La componente horizontal del campo vectorial tiene dirección negativa para $u \in[0,1]$.

$$
\frac{d u}{d \tau}=-v<0 .
$$

Cualquiera sea el signo de $\frac{d v}{d \tau}=B\left(1-C+N v^{\sigma}\right) v$, se obtiene que toda solución ingresa a $\bar{\Gamma}$ y no puede salir de allí.

\section{Observación 3.1.}

1. Asumiendo que $u=0$, se observa que la segunda ecuación del sistema (2.2) queda

$$
\frac{d v}{d \tau}=B\left(-C+N v^{\sigma}\right) v .
$$

Claramente, $\frac{d v}{d \tau}>0$, si y sólo si, $-C+N v^{\sigma}>0$.

Esta propiedad implica que existen soluciones que no son acotadas.

2. Ecológicamente, el ítem 1, significa que en ausencia de su presa preferida, el crecimiento de la población de depredadores no tiene límite.

Este comportamiento puede interpretarse asumiendo que el depredador es generalista; en ausencia de su presa favorita, el depredador busca un alimento alternativo.

La extinción de las presas puede ser de interés, si esta población constituye una plaga.

3. Hay una región triangular definida por

$$
T=\{(u, v) \in \bar{\Gamma}: 0 \leq u \leq 1,0 \leq v \leq 1-u\}
$$

que es relevante en términos de dinámica.

Si $v>1-u$, se tiene que $\frac{d u}{d \tau}<0$, implicando que las soluciones podrían entrar a la región $T$. Eso dependerá del signo de la segunda ecuación del sistema $\frac{d v}{d \tau}=B\left(u-C+N v^{\sigma}\right) v$.

3.1. Naturaleza de los puntos de equilibrio. El sistema (2.2) tiene las siguientes propiedades:

Proposición 3.2. Naturaleza de los puntos de equilibrio sobre el eje horizontal.

1. El punto de equilibrio $(0,0)$ es una silla hiperbólica para todo valor de parámetros.

2. El punto $(1,0)$ es.

i) una silla hiperbólica, si y sólo si, $C<1$,

ii) nodo atractor, si y sólo si, $C>1$,

iii) atractor no-hiperbólico, si y sólo si, $C=1$.

Demostración: Es inmediata, usando la matriz Jacobiana de la linealización (Teorema de HartmanGrobman en los equilibrios hiperbólicos [6]). Las respectivas matrices Jacobianas evaluadas en cada punto son

$$
D Y_{\mu}(0,0)=\left(\begin{array}{cc}
1 & 0 \\
0 & -B C
\end{array}\right),
$$




$$
D Y_{\mu}(1,0)=\left(\begin{array}{cc}
-1 & -1 \\
0 & B(1-C)
\end{array}\right) \text {. }
$$

De acuerdo al Teorema de la traza y el determinante [20], se tiene el enunciado

Proposición 3.3. Naturaleza del punto de equilibrio sobre el eje vertical

El punto de equilibrio $\left(0,\left(\frac{C}{N}\right)^{\frac{1}{\sigma}}\right)$ es

i) Un punto silla hiperbólica, si y sólo si, $C>N$.

ii) Un punto silla-no-hiperbólico, si y sólo si, $C=N$.

iii) Un nodo repulsor hiperbólico, si y sólo si, $C<N$.

Demostración: La matriz Jacobiana evaluada en $\left(0,\left(\frac{C}{N}\right)^{\frac{1}{\sigma}}\right)$ es

Tenemos que

$$
D Y_{\mu}\left(0,\left(\frac{C}{N}\right)^{\frac{1}{\sigma}}\right)=\left(\begin{array}{cc}
1-\left(\frac{C}{N}\right)^{\frac{1}{\sigma}} & 0 \\
B\left(\frac{C}{N}\right)^{\frac{1}{\sigma}} & B C \sigma
\end{array}\right) .
$$

$$
\operatorname{det} D Y_{\mu}\left(0,\left(\frac{C}{N}\right)^{\frac{1}{\sigma}}\right)=B C \sigma\left(1-\left(\frac{C}{N}\right)^{\frac{1}{\sigma}}\right),
$$

cuyo signo depende del factor $D Y_{\mu}\left(0,\left(\frac{C}{N}\right)^{\frac{1}{\sigma}}\right)_{11}=T_{0}=1-\left(\frac{C}{N}\right)^{\frac{1}{\sigma}}$.

Si $T_{0}<0$, implica que $C>N$, y el equilibrio $\left(0,\left(\frac{C}{N}\right)^{\frac{1}{\sigma}}\right)$ es punto silla hiperbólica.

Si $T_{0}>0$, implica que $C<N$, y la naturaleza del equilibrio $\left(0,\left(\frac{C}{N}\right)^{\frac{1}{\sigma}}\right)$ depende de la

$$
\operatorname{tr} D Y_{\mu}\left(0,\left(\frac{C}{N}\right)^{\frac{1}{\sigma}}\right)=1-\left(\frac{C}{N}\right)^{\frac{1}{\sigma}}+B C \sigma,
$$

la cual es siempre positiva.

Por lo tanto, el punto $\left(0,\left(\frac{C}{N}\right)^{\frac{1}{\sigma}}\right)$ es nodo repulsor.

Proposición 3.4. Naturaleza del único punto de equilibrio positivo.

Suponiendo que $\left(u_{e}, v_{e}\right)$ existe en el primer cuadrante, la abscisa del punto de equilibrio es $u_{e}<C$. Supongamos que i) $1>C$ y $\frac{C}{N}>1$.

El punto de equilibrio positivo $\left(u_{e}, v_{e}\right)$ es

i) Un atractor hiperbólico, si y sólo si, $B<\frac{u_{e}}{\sigma\left(C-u_{e}\right)}$,

ii) Un repulsor hiperbólico, si y sólo si, $B>\frac{u_{e}}{\sigma\left(C-u_{e}\right)}$, rodeado de un ciclo límite estable.

iii) Un foco débil, si y sólo si, $B=\frac{u_{e}}{\sigma\left(C-u_{e}\right)}$.

Supongamos que ii) $1<C$ y $\frac{C}{N}<1$.

El punto de equilibrio positivo $\left(u_{e}, v_{e}\right)$ es silla hiperbólica.

Demostración: La matriz Jacobiana del sistema (linealización) en el punto $\left(u_{e}, 1-u_{e}\right)$ es:

$$
D Y_{\mu}\left(u_{e}, 1-u_{e}\right)=\left(\begin{array}{cc}
-u_{e} & -u_{e} \\
B\left(1-u_{e}\right) & B\left(u_{e}-C+(1+\sigma) N\left(1-u_{e}\right)^{\sigma}\right)
\end{array}\right),
$$

De la ecuación (2.3) se obtiene que $C-u_{e}=N\left(1-u_{e}\right)^{\sigma}$.

Por lo tanto,

obteniendo que

$$
D Y_{\mu}\left(u_{e}, 1-u_{e}\right)=\left(\begin{array}{cc}
-u_{e} & -u_{e} \\
B\left(1-u_{e}\right) & B \sigma\left(C-u_{e}\right)
\end{array}\right)
$$

$$
\operatorname{det} D Y_{\mu}\left(u_{e}, 1-u_{e}\right)=-B u_{e}\left(u_{e}+C \sigma-\sigma u_{e}-1\right)
$$

Además, el intercepto de la isoclina de los depredadores es mayor que la ordenada del punto de equilibrio positivo, cuando existen es decir, $\frac{C}{N}>1-u_{e}>0$.

i) Si $1>C$ y $\frac{C}{N}>1$, se cumple que $1>C>u_{e}$.

Luego, $1-u_{e}>C-u_{e}>\sigma\left(C-u_{e}\right)$, $\operatorname{con} \sigma<1$.

Obteniendo que

$$
\operatorname{det} D Y_{\mu}\left(u_{e}, 1-u_{e}\right)=-B u_{e}\left(\sigma\left(C-u_{e}\right)-\left(1-u_{e}\right)\right)>0,
$$

y la naturaleza del punto de equilibrio positivo depende del signo de la traza, dada por $\operatorname{tr} D Y_{\mu}\left(u_{e}, 1-u_{e}\right)=-u_{e}+B \sigma\left(C-u_{e}\right)$.

ii) Cuando $1<C, \frac{C}{N}<1, C>u_{e}$ y $\frac{C}{N}>1-u_{e}>0$, se tiene que $C>1>\frac{C}{N}>1-u_{e}$

Como $\left(1-u_{e}\right)>\sigma\left(1-u_{e}\right)$, con $\sigma<1$, se tiene que $\sigma\left(C-u_{e}\right)-\left(1-u_{e}\right)>\sigma\left(C-u_{e}\right)-\sigma\left(1-u_{e}\right)=\sigma(C-1)>0$. 
Así obtenemos que:

$\operatorname{det} D Y_{\mu}\left(u_{e}, 1-u_{e}\right)=-B u_{e}\left(\sigma\left(C-u_{e}\right)-\left(1-u_{e}\right)\right)<0$,

y el punto de equilibrio positivo es una silla hiperbólica.

\section{Corolario 3.1.}

Existe al menos un ciclo límite alrededor del punto $\left(x_{e}, 1-u_{e}\right)$.

Demostración: Cuando el punto $\left(x_{e}, 1-u_{e}\right)$ es repulsor, de acuerdo con el teorema de PoincaréBendixson en la región $\bar{\Gamma}$, debe existir un ciclo límite.

Es inmediata pues la condición de transversalidad [6] implica que

$$
\frac{\partial}{\partial B}\left(\operatorname{tr} D Y_{\mu}\left(u_{e}, 1-u_{e}\right)\right)=\frac{\partial}{\partial B}\left(-u_{e}+B \sigma\left(C-u_{e}\right)\right)=\sigma\left(C-u_{e}\right)<0 .
$$

4. Conclusiones. Con el análisis realizado en este trabajo ampliamos los resultados obtenidos para el sistema analizado parcialmente en [3], cuando $0<\sigma<1$. Entre otros resultados, es posible probar, la existencia de subconjuntos en el espacio de parámetros para los cuales:

i) La población de depredadores tiende a un tamaño infinito, mientras que la población de presas se extingue.

ii) Hay un único punto de equilibrio positivo en el plano de fase.

iii) Una bifurcación de Hopf es generada en el punto de equilibrio positivo dando origen a un ciclo límite estable.

iv) Queda en evidencia que las soluciones del sistema son altamente sensibles a las condiciones iniciales, pues dos trayectorias muy cercanas pueden tener $\omega$-límites muy alejados.

v) Coexisten dos atractores por lo cual se genera el fenómeno de bi-estabilidad.

Hemos mostrado que la incorporación del comportamiento social de los depredadores para optimizar la captura de sus presas influencia fuertemente en las propiedades matemáticas del sistema EDO que describe el modelo. Este sistema tiene dinámicas más ricas e interesantes que el modelo de Volterra [25] en el cual nos hemos basado para incluir la colaboración o cooperación entre los depredadores.

Entonces surge la necesidad discutir acerca de la validez del modelo planteado. Esto es debido a que para las mismos valores de parámetros, pero con diferentes condiciones iniciales, las poblaciones pueden coexistir periodicamente o en valores fijos de los tamaños poblacionales, o bien, la población de presas desaparece y la población de depredadores alcanza su máximo tamaño.

\section{Tareas pendientes}

- Determinar la debilidad del foco débil o probar la existencia de un único ciclo límite en el sistema.

- Determinar un subconjunto en el espacio de parámetros para los cuales existe una curva separatriz.

- Realizar un diagrama de bifurcaciones uniparamétrico para estudiar la sensibilidad del parámetro de cooperación $\sigma$.

- Establecer las bifurcaciones globales del sistema.

Perspectivas futuras

En base al modelo estudiado podemos derivar nuevos modelos de depredación, en los cuales se asuman los siguientes fenómenos ecológicos:

- La población de presas es afectada por un efecto Allee [7, 17, 24, 26].

- Una fracción de la población de presas hace uso de refugio [11,21].

- La acción del depredador es descrita por una respuesta funcional no-lineal [22, 23].

- La colaboración entre los depredadores es saturada [13].

ORCID and License

Eduardo González-Olivares https:// orcid.org/0000-0003-3907-0076

Alejandro Rojas-Palma https://orcid.org/0000-0002-5837-1571

This work is licensed under the Creative Commons - Attribution 4.0 International (CC BY 4.0)

\section{Referencias}

[1] Antonelli PL, Kazarinoff ND. Starfish predation of a growing coral reef community. J. Theor. Biol. 1984;107:667-684.

[2] Antonelli PL, Kazarinoff ND. Modelling density-dependent aggregation and reproduction in certain terrestrial and marine ecosystems: A comparative study, Ecological Modelling 1988;41:219-227.

[3] Antonelli PL, Lin X. Bifurcation analysis on a coral-starfish model. Mathematical and Computer Modelling 13 (1990) $35-44$.

[4] Bazykin AD. Nonlinear Dynamics of interacting populations, World Scientific Publishing Co. Pte. Ltd., Singapore 1998.

[5] Berryman AA, Gutierrez AP, Arditi R. Credible, parsimonious and useful predator-prey models - A reply to Abrams, Gleeson and Sarnelle, Ecology. 1995 76:1980-1985.

[6] Chicone C. Ordinary differential equations with applications (2nd edition), Texts in Applied Mathematics 34, Springer 2006.

[7] Courchamp F, Berec L, Gascoigne J. Allee effects in Ecology and Conservation, Oxford University Press 2007.

[8] Freedman HI. Deterministic Mathematical Model in Population Ecology, Marcel Dekker 1980. 
[9] Gause GF. The struggle for existence, Dover 1934.

[10] Goh B-S. Management and Analysis of Biological Populations, Elsevier Scientific Publishing Company, 1980.

[11] González-Olivares E, González-Yañez B, Becerra-Klix R. Prey refuge use as a function of predator-prey encounters, Private communication, submitted to International Journal of Biomathematics (2012).

[12] González-Olivares E, Cabrera-Villegas J, Córdova-Lepe F, Rojas-Palma A. Competition among predators and Allee effect on prey: their influence on a Gause-type predation model, Mathematical Problems in Engineering, vol. 2019, Article ID 3967408, 19 pages, 2019.

[13] González-Olivares E, Valenzuela-Figueroa S, Rojas-Palma A. A simple Gause type predator-prey model considering social predation, Mathematical Methods in the Applied Sciences 42 (2019) 5668-5686.

[14] González-Olivares E, Rojas-Palma A. Influencia del efecto Allee fuerte en las presas y de la competición entre los depredadores en modelos de depredación del tipo Leslie-Gower, Selecciones Matemáticas; 2020; 7(2):302-313.

[15] González-Olivares E, Rojas-Palma A. Estabilidad en sistemas cuadráticos del tipo Kolmogorov describiendo interacciones entre dos especies. Una breve revisión, Selecciones Matemáticas. 2021; 8(1):132-146.

[16] Hilker FM, Paliaga M, Venturino E. Diseased social predators, Bulletin of Mathematical Biology 2017;79:2175-2196.

[17] Jang SR-J, Zhang W, Larriva V. Cooperative hunting in a predator-prey system with Allee effects in the prey, Natural Resource Modelling 31 (2018) 1-20 pp.

[18] Kot M. Elements of Mathematical Ecology, Cambridge University Press, Cambridge, New York, 2003.

[19] MacNulty DR, Tallian A, Stahler DR, Smith DW. Influence of group size on the success of wolves hunting bison, Plos One 2014;9(11):e112884 1-8.

[20] May RM. Stability and complexity in model ecosystems (2nd edition), Princeton University Press, 2001.

[21] Maynard-Smith J. Models in Ecology, University Press 1974.

[22] Rojas-Palma A, González-Olivares E. Gause type predator-prey models with a generalized rational non-monotonic functional response, In J. Vigo-Aguiar (Ed.) Proceedings of the 14th International Conference on Computational and Mathematical Methods in Science and Engineering, CMMSE 2014, Volume 4 (2014) 1092-1103. ISBN: 978-84-616-9216-3.

[23] Taylor RJ. Predation, Chapman and Hall, 1984.

[24] Teixeira Alves M, Hilker FM. Hunting cooperation and Allee effects in predators, J. of Theoretical Biology 419 (2017) 13-22.

[25] Turchin P. Complex Population Dynamics: A Theoretical/Empirical Synthesis, Princeton University Press, Princeton, New Jersey, 2003.

[26] Ye P, Wu D. Impacts of strong Allee effect and hunting cooperation for a Leslie-Gower predator-prey system, Chinese J. of Physics 68 (2020) 49-64. 